\title{
Continuous cellular automata on irregular tessellations: mimicking steady-state heat flow
}

\author{
Jan M. Baetens and Bernard De Baets \\ KERMIT \\ Ghent University \\ Coupure links 653, 9000 Gent, Belgium \\ Email: \{jan.baetens,bernard.debaets\}@ugent.be
}

\begin{abstract}
Leaving a few exceptions aside, cellular automata (CA) and the intimately related coupled-map lattices (CML), commonly known as continuous cellular automata (CCA), as well as models that are based upon one of these paradigms, employ a regular tessellation of an Euclidean space in spite of the various drawbacks this kind of tessellation entails, such as its inability to cover surfaces with an intricate geometry, or the anisotropy it causes in the simulation results. Recently, a CCA-based model describing steady-state heat flow has been proposed as an alternative to Laplace's equation that is, among other things, commonly used to describe this process, yet, also this model suffers from the aforementioned drawbacks since it is based on the classical CCA paradigm. To overcome these problems, we first conceive CCA on irregular tessellations of an Euclidean space after which we show how the presented approach allows for a straightforward simulation of steady-state heat flow on surfaces with an intricate geometry, and, as such, constitutes a full-fledged alternative for the commonly used and easy-toimplement finite difference method, and the more intricate finite element method.
\end{abstract}

Index Terms-cellular automata; coupled-map lattice; irregular tessellations; heat flow

\section{INTRODUCTION}

Ever since the publication of Conway's 'Game of Life' [1], cellular automata (CA), originally conceived by von Neumann in the early 1950s [2], and the closely related coupled-map lattices (CML) [3], also known as continuous cellular automata (CCA), have aroused numerous researchers' interest, which comes forward by the growing number of publications on these topics. In fundamental sciences, much attention has been paid to the dynamical properties of (C)CA [4], [5], [3], [6], whereas many sound (C)CA-based models have been developed in various scientific disciplines, such as epidemiology [7], [8], fire science [9], [10], [11], geography [12], (micro)biology [13], [14], [15], medical science [16], [17], and transportation [18], [19]. A comprehensive review of the (C)CA-based models that have been developed recently in geomorphology can be found in [20].

Nonetheless a few recent studies have highlighted CA on other regular tessellations of the Euclidean plane [11], [21], [22], most of the above-mentioned studies use a square tessellation of the two-dimensional Euclidean space owing to its implementational simplicity and computational efficiency [23]. Although such a tessellation allows for a thorough study of a (C)CA's dynamical properties, it suffers from several drawbacks hampering the widespread use of (C)CA-based models as full-fledged alternatives of well-established models based upon partial differential equations. First, by limiting the discretization of the spatial domain to regular $n$-polytopes, the applicability of (C)CA-based models becomes restricted to spatial domains with a rather simple geometry that imposes even elementary boundary conditions [24], [25]. Second, a square tessellation artificially restricts the number of possible flow directions [22] in (C)CA-based mass flow models that are getting commonly used in hydrological and geological studies [26], [27], [28], [29]. Third, the use of a regular tessellation often results in artificially anisotropic patterns [30]. Fourth, the classical (C)CA paradigm hinders the use of spatio-temporal data in vector format, which is built up from a set of geometric primitives, as well as the integration of the (C)CA concept in existing, vector-based geographical information systems software. Fifth, it can be questioned whether (C)CA based upon a regular tessellation give rise to possible side-effects on the simulation results as a consequence of the regularity involved in its topology [31], [32], [33].

In order to overcome these barriers, (C)CA on irregular tessellations of $\mathbb{R}^{n}$ can be conceived. Such tessellations can be obtained easily by relying on a rich variety of already well-established tessellation methods, such as the Delaunay tessellation, which are often built-in in mathematical software packages or are distributed free of charge as add-ons. By means of this paper, in which we formalize CCA on irregular tessellations and illustrate their usability, we hope to instigate the development of CCA-based models that benefit from weakening the topology constraint that is adhered to by the classical CCA paradigm.

In Section II we briefly present the mathematical formalization of CCA on irregular tessellations, while their graph representation is addressed in Section III. In the ultimate section of this paper we illustrate the usability and strengths of the irregular CCA paradigm for describing steady-state heat flow.

\section{CONTINUOUS CELlular AUtOMATA ON IRREGUlAR TESSELLATIONS}

\section{A. Definition}

First, we state the definition of a CCA on an arbitrary tessellation of an $n$-dimensional Euclidean space, which con- 
stitutes an extension to the classical CCA paradigm that predominantly relies on a square or cuboid tessellation of $\mathbb{R}^{2}$ or $\mathbb{R}^{3}$, respectively.

Definition 2.1: A continuous cellular automaton $(C C A) \mathcal{C}$ can be represented as a sextuple

$$
\mathcal{C}=\left\langle\mathcal{T}, S, s, s_{0}, N, \Phi\right\rangle,
$$

where

(i) $\mathcal{T}$ is a countably infinite tessellation of an $n$-dimensional Euclidean space $\mathbb{R}^{n}$, consisting of non-overlapping cells $c_{i}, i \in \mathbb{N}$, that allows for a complete coverage of $\mathbb{R}^{n}$.

(ii) $S$ is an infinite set of states, often $S \subseteq \mathbb{R}$.

(iii) The output function $s: \mathcal{T} \times \mathbb{N} \rightarrow S$ yields the state value of cell $c_{i}$ at the $t$-th discrete time step, i.e. $s\left(c_{i}, t\right)$.

(iv) The function $s_{0}: \mathcal{T} \rightarrow S$ assigns to every cell $c_{j}$ an initial state, i.e. $s\left(c_{i}, 0\right)=s_{0}\left(c_{i}\right)$.

(v) The neighborhood function $N: \mathcal{T} \rightarrow \bigcup_{p=1}^{\infty} \mathcal{T}^{p}$ maps every cell $c_{i}$ to a finite sequence $N\left(c_{i}\right)=\left(c_{i_{j}}\right)_{j=1}^{\left|N\left(c_{i}\right)\right|}$, consisting of $\left|N\left(c_{i}\right)\right|$ distinct cells $c_{i_{k}}$.

(vi) $\Phi=\left(\phi_{i}\right)_{i \in \mathbb{N}}$ is a family of functions

$$
\phi_{i}: S^{\left|N\left(c_{i}\right)\right|} \rightarrow S
$$

each $\phi_{i}$ governing the dynamics of cell $c_{i}$, i.e.

$$
\begin{aligned}
& \qquad s\left(c_{i}, t+1\right)=\phi_{i}\left(\tilde{s}\left(N\left(c_{i}\right), t\right)\right), \\
& \text { where } \tilde{s}\left(N\left(c_{i}\right), t\right)=\left(s\left(c_{i_{j}}, t\right)\right)_{j=1}^{\left|N\left(c_{i}\right)\right|} .
\end{aligned}
$$

Since the formulation of premises (i), (v) and (vi) in this definition differs from the original paradigm as proposed by Kaneko [3], we elaborate on them in the remainder of this section.

\section{B. Tessellations of $\mathbb{R}^{2}$}

Seen the fact that the CCA-based models presented in the subsequent sections describe phenomena occurring in the Euclidean plane, we restrict the discussion to tessellations of $\mathbb{R}^{2}$. For that purpose, we consider a countably infinite, irregular tessellation $\mathcal{T}$ of the Euclidean plane, which can be envisaged as a set $\mathcal{P}^{2}=\left\{p_{1}^{2}, p_{2}^{2}, \ldots, p_{i}^{2}, \ldots\right\}$ of convex 2-polytopes, i.e. polygons, $p_{i}^{2}$, which, on their turn, can be assembled from a set of line segments $p_{j}^{1}$ that can be denoted as

$$
\mathcal{P}_{i}^{1}=\left\{p_{i_{1}}^{1}, p_{i_{2}}^{1}, \ldots, p_{i_{l_{i}^{1}}}^{1}\right\},
$$

where $l_{i}^{1}$ indicates the number of line segments needed to construct $p_{i}^{2}$. Sticking to this notation, the set of line segments needed to construct all $p_{i}^{2}$ in $\mathcal{T}$ is then given by $\mathcal{S}=\bigcup \mathcal{P}_{i}^{1}$. Figure 1(b) visualizes this construction for a convex polygon $p_{i}^{2}$ belonging to a tessellation $\mathcal{T}$ of $\mathbb{R}^{2}$. Clearly, every line segment $p_{j}^{1}$ in $\mathcal{P}_{i}^{1}$ can be formed by connecting two points in $\mathbb{R}^{2}$ such that

$$
\mathcal{P}_{i}^{0}=\left\{p_{i_{1}}^{0}, p_{i_{2}}^{0}, \ldots, p_{i_{l_{i}^{0}}}^{0}\right\},
$$

constitutes the set of vertices $p_{j}^{0}$ from which $p_{i}^{2}$ can be assembled (Fig. 1(c)), whereas $\mathcal{E}=\bigcup_{j} \mathcal{P}_{j}^{0}$ is the set of endpoints necessary to construct all line segments in $\mathcal{T}$.

In case one is faced with an irregular tessellation of a 3- or higher-dimensional Euclidean space, the above-outlined line of reasoning can be extended by envisaging its tessellation as a set $\mathcal{P}^{n}=\left\{p_{1}^{n}, p_{2}^{n}, \ldots, p_{i}^{n}, \ldots\right\}$ of convex $n$-polytopes that are built up from $(n-1)$-polytopes, which can be further decomposed in $(n-2),(n-3), \ldots, 0$-polytopes.

\section{The neighborhood function $N$}

In order to streamline the construction of the sequence $\left(c_{i_{j}}\right)_{j=1}^{\left|N\left(c_{i}\right)\right|}$, we first introduce the intuitive concept of socalled $m$-dimensional contact.

Definition 2.2: Two n-polytopes $p_{i}^{n}, p_{j}^{n} \in \mathcal{T}$ have $m$ dimensional contact, with $m \leq n$, if they share at least one m-polytope.

Clearly, it follows that $m$-dimensional contact between $n$ polytopes implies $0,1, \ldots,(m-1)$-dimensional contact. The former definition can now be used to unambiguously set up a subset $\mathcal{N}_{i} \subset \mathcal{T}$, further referred to as a topological neighborhood, containing those cells $c_{j} \in \mathcal{T}$ that are considered $c_{i}$ 's neighbors.

Definition 2.3: The $m$-th order topological neighborhood $\mathcal{N}_{i}^{m}$ of an n-polytope $p_{i}^{n} \in \mathcal{T}$, with $0 \leq m<n$, contains all n-polytopes $p_{j}^{n} \in \mathcal{T}$ that have $m$-dimensional contact with $p_{i}^{n}$, i.e. $\mathcal{N}_{i}^{m}=\left\{p_{j}^{n} \in \mathcal{T} \mid \mathcal{P}_{i}^{m} \cap \mathcal{P}_{j}^{m} \neq \emptyset\right\}$.

If tessellations of $\mathbb{R}^{2}$ are considered, Definition 2.3 allows the construction of either a 0-th or 1-st order topological neighborhood, which are better known as the Moore and von Neumann neighborhood, respectively. Both neighborhoods are illustrated in Fig. 2 for a convex polygon $c_{1}$ in an irregular tessellation of $\mathbb{R}^{2}$. It should be clear that the geometric discrepancies existing between cells in $\mathcal{T}$, which can be traced back to differences between the position of the $n$-polytopes' centroids, and, the polygons' circumference and area are not taken into account for pinning down a cell's topological neighborhood. Obviously, these geometric characteristics could be used to exclude cells not fulfilling certain criteria from the topological neighborhoods, and, as such, give rise to a restricted topological neighborhood $\mathcal{N}_{i}^{m, R}$. For instance, if tessellations of $\mathbb{R}^{2}$ are at stake, a threshold can be imposed on the minimum proportion of $c_{i}$ 's circumference that must be shared with $c_{j}$ so that $c_{j}$ is still considered $c_{i}$ 's neighbor. Figure 2(c) shows the restricted topological neighborhood of a convex polygon $c_{1}$ enclosing those cells $c_{j}$ belonging to $\mathcal{N}_{1}^{1}$ that make up at least $20 \%$ of $c_{1}$ 's circumference. Unless stated otherwise, a Moore neighborhood is used throughout the remainder of this paper.

\section{Transition function $\phi_{i}$}

According to Def. 2.1 every $c_{i}$ in $\mathcal{T}$ has its distinct transition function $\phi_{i}$ governing its dynamics. Yet, we can contemplate the family of CA for which $\phi_{i}=\Theta$ is the same for all $c_{i} \in \mathcal{T}$. 


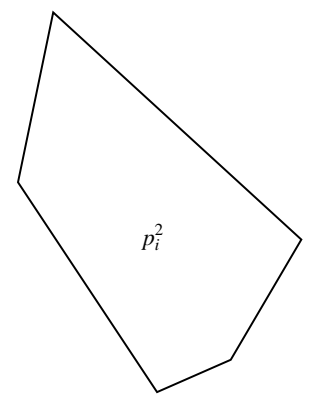

(a)

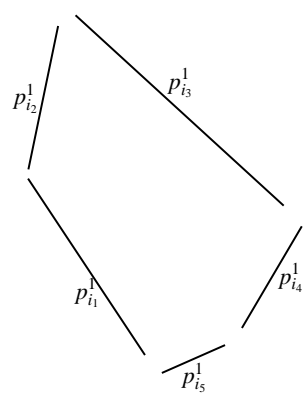

(b)

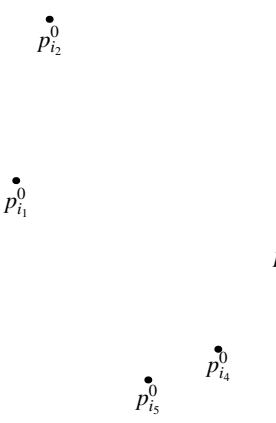

(c)

Fig. 1. Assembly of vertices $p_{i_{j}}^{0}$ in $\mathbb{R}^{2}$ leading to line segments $p_{i_{k}}^{1}$ that on their turn can be joined to form the polygon $p_{i}^{2}$.

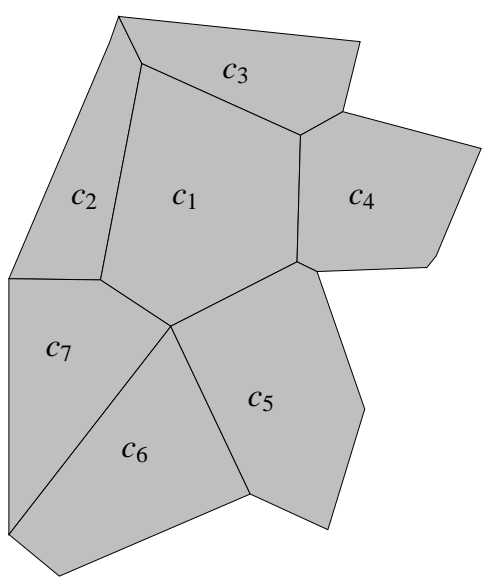

(a)

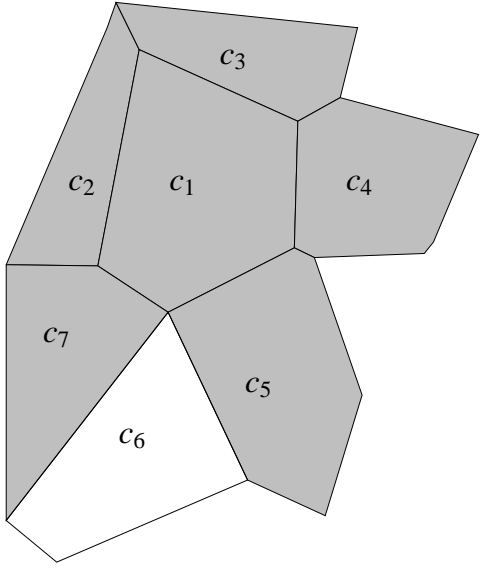

(b)

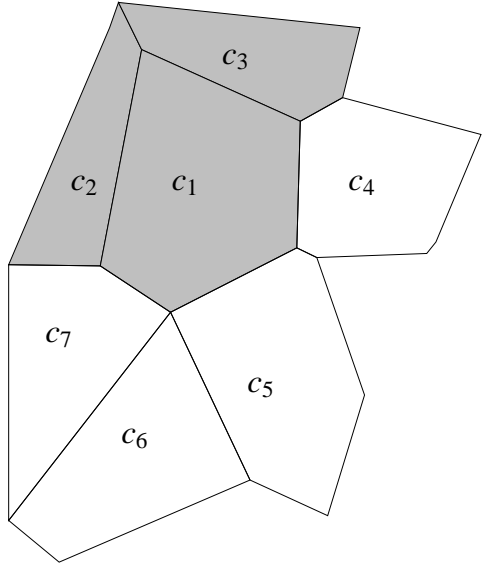

(c)

Fig. 2. Moore (a), von Neumann (b) and (c) restricted topological neighborhood of a convex polygon $c_{1}$.

Definition 2.4: A homogeneous continuous cellular automaton (CCA) is a CCA fulfilling premises ( $i)-(v)$ of Definition 2.1 and for which there exists $a \Theta: \bigcup_{k \in \mathbb{N}} S^{k} \rightarrow S$ such that

$$
s\left(c_{i}, t+1\right)=\Theta\left(\tilde{s}\left(N\left(c_{i}\right), t\right)\right) .
$$

Essentially, most works on CCA merely concern homogeneous CCA [34], [35].

\section{GRAPH REPRESENTATION OF CCA ON IRREGUlAR TESSELLATIONS}

In order to simplify their implementation and to elucidate the irregular CCA paradigm, a graph representation of CCA on irregular tessellations of $\mathbb{R}^{n}$ can be exploited. If a topological neighborhood is considered (Cfr. Def. 2.3), $\mathcal{T}$ is mapped on an undirected graph $G(V, E)$, with vertex set $V=\mathcal{T}$ and $E$ representing the edge set of $G$, assembled in accordance with Definition 2.3. Hence, an edge $e_{i j}$ exists between two vertices $c_{i}$ and $c_{j}$ if $c_{j} \in N\left(c_{i}\right)$. On the other hand, if the geometric characteristics of $c_{i}$ must be taken into account when constructing $N\left(c_{i}\right)$, a directed graph $G_{d}\left(V, E_{d}\right)$ should be employed, since $c_{j} \in \mathcal{N}_{i}^{m, R}$ does not necessarily imply that $c_{j} \in \mathcal{N}_{i}^{m, R}$.

Figure 3(a) shows a finite 2D tessellation of a compact subset of $\mathbb{R}^{2}$ consisting of 15 irregular polygons, while its projection onto an undirected graph $G$ is given in Fig. 3(b). Dashed edges only exist if a Moore neighborhood is at stake, since a von Neumann neighborhood discards polygons that have 0-dimensional contact. Hence, if the latter is at stake only the non-dashed edges are present.

\section{Modeling STEAdY-STATE HEAT Flow}

A. A generalized CCA-based model for steady-state heat flow

As an exemplary diffusion process, we consider in this section the steady-state flow of heat through a two-dimensional rectangular surface $S \subset \mathbb{R}^{2}$ that is commonly addressed using Laplace's equation:

$$
\nabla^{2} T(x, y)=0
$$

where $T(x, y)$ denotes the steady-state temperature expressed in degrees centigrade at every point $(x, y)$ in $S$.

For setting up a CCA-based model describing steady-state heat flow, which is necessarily based upon discrete spatial entities (i.e. cells $c_{i}$ ), it can be reasoned that if such a cell is surrounded by a given number of neighboring cells, each maintained at constant temperature, it will reach a steadystate temperature after a sufficient amount of time that is completely determined by the temperature of its neighbors. 


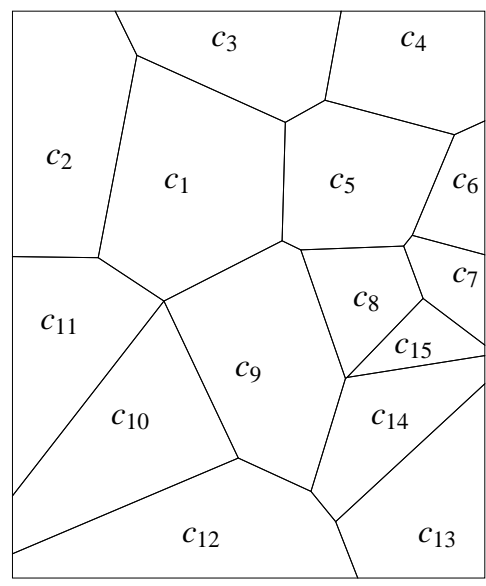

(a)

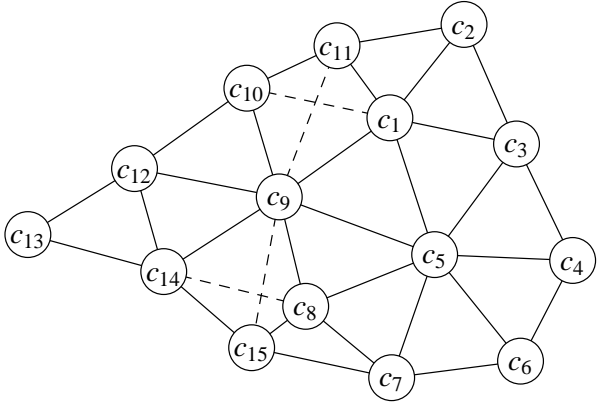

(b)

Fig. 3. Representation of a discrete irregular tessellation of a compact subset of $\mathbb{R}^{2}$ (a) and the graph $G$ obtained after mapping this tessellation on $G$ (b). Dashed vertices only exist when a Moore neighborhood is considered.

More specifically, this steady-state temperature will be the average value of the temperatures at which the neighboring cells are maintained. Hence, if we discard the difference in the length of the common line segment through which neighboring cells exchange heat, and which unavoidably exists between cells in an irregular tessellation of $\mathbb{R}^{2}$, the steady-state temperature in the cells of $\mathcal{T}$ can be obtained by iteratively evaluating

$$
W\left(c_{i}, t+1\right)=\Theta\left(\tilde{s}\left(N\left(c_{i}\right), t\right)\right)=\frac{\sum_{c_{j} \in N\left(c_{i}\right)} W\left(c_{j}, t\right)}{\left|N\left(c_{i}\right)\right|},
$$

for all $c_{i}$, until $\left|W\left(c_{i}, t+1\right)-W\left(c_{i}, t\right)\right| \leq \phi, \forall c_{i} \in \mathcal{T}$, where $\phi$ is a given halting criterion and $W\left(c_{i}, t\right)$ denotes the temperature expressed in degrees centigrade in cell $c_{i}$ at the $t$-th time step. In contrast with $T(x, y), W\left(c_{i}, t\right)$ constitutes a discrete function since it yields a value for discrete cells and time steps only. Taking into account the discrepancies between the length of the exchange 'surface', Eq. (2) can be modified to:

$W\left(c_{i}, t+1\right)=\Theta\left(\tilde{s}\left(N\left(c_{i}\right), t\right)\right)=\frac{1}{C_{i i}} \sum_{c_{j} \in N\left(c_{i}\right)} W\left(c_{j}, t\right) C_{i j}$,

where $C_{i j}$ denotes the length of the line segment that is shared by $c_{i}$ and $c_{j}$. It should be clear that Eqs. (2) and (3) constitute the transition function of a homogeneous CCA $\mathcal{C}=\left\langle\mathcal{T}, S, s, s_{0}, N, \Theta\right\rangle$, with $\mathcal{T}=\left\{c_{1}, c_{2}, \ldots\right\}, S=\mathbb{R}$, $s\left(c_{i}, t\right)=W\left(c_{i}, t\right), s_{0}\left(c_{i}\right)=W\left(c_{i}, 0\right), N\left(c_{i}\right)=\mathcal{N}_{i}^{0}$, and $\Theta$ being the right-hand-side of Eq. (2) or (3). Essentially, Eqs. (2) and Eq. (3) constitute an extension to the CCA proposed by Schiff [36] for describing heat flow on a square tessellation that suffers from several drawbacks as discussed in Section I.

\section{$B$. Assessing the validity of the CCA-based model}

In order to demonstrate the validity of Eq. (2), we consider the heat flow in a square plate, which is subject to the boundary conditions

$$
\begin{array}{ll}
T(0, y)=0, & 0<y<s, \\
T(s, y)=0, & 0<y<s, \\
T(x, 0)=T_{b}, & 0 \leq x \leq s, \\
T(x, s)=T_{t}, & 0 \leq x \leq s,
\end{array}
$$

where $s$ is the side length of the plate. Since an analytical solution can be found for this boundary value problem a sound assessment of Eq. (2)'s validity becomes possible. The latter solution can be found straightforwardly by separation of variables, and can be simplified to

$$
\begin{array}{r}
T(x, y)=4 T_{t} \sum_{m=1}^{+\infty} \frac{\sinh \left(m^{*} y s^{-1}\right) \sin \left(m^{*} x s^{-1}\right)}{m^{*} \sinh \left(m^{*}\right)}+ \\
4 T_{b} \sum_{m=1}^{+\infty} \frac{\sinh \left(m^{*}(s-y) s^{-1}\right) \sin \left(m^{*} x s^{-1}\right)}{m^{*} \sinh \left(m^{*}\right)}
\end{array}
$$

where $m^{*}=(2 m-1) \pi$ was introduced for reasons of clarity. In the remainder of this subsection, we consider a steel plate with side length $s=1, T_{t}=50$ and $T_{b}=50$. The simulations with the CCA-based model were performed using a regular tessellation consisting of $100 \times 100$ square cells and a value of $10^{-7}$ was selected for $\phi$, while 200 terms of the series in Eq. (4) were taken into account. Figure 4, depicting the steady-state temperature distribution along three horizontal cross-sections obtained using the analytical solution (Eq. (4)) on the one hand, and the CCA-based model (Eq. (2)) on the other hand, and Fig. 5 visualizing the absolute difference between $W\left(c_{i}, t_{\phi}\right)$ and the corresponding $T\left(x_{i}^{g}, y_{i}^{g}\right)$, where $t_{\phi}$ is the number of time steps needed such that $\left|W\left(c_{i}, t+1\right)-W\left(c_{i}, t\right)\right| \leq \phi, \forall c_{i} \in \mathcal{T}$ and $\left(x_{i}^{g}, y_{i}^{g}\right)$ represents $c_{i}$ 's center of gravity, clearly show the close agreement between the simulated temperature distributions obtained with both approaches. The good agreement between 
TABLE I

AVERAGE ABSOLUTE DIFFERENCE $\mu_{e}$ BETWEEN $W\left(c_{i}, t_{\phi}\right)$, WHERE $t_{\phi}$ IS THE NUMBER OF TIME STEPS NEEDED SUCH THAT

$\left|W\left(c_{i}, t+1\right)-W\left(c_{i}, t\right)\right| \leq \phi, \forall c_{i} \in \mathcal{T}$, AND THE CORRESPONDING $T\left(x_{i}^{g}, y_{i}^{g}\right)$, WHERE $\left(x_{i}^{g}, y_{i}^{g}\right)$ REPRESENTS $c_{i}$ 'S CENTER OF GRAVITY, TOGETHER WITH $t_{\phi}$.

\begin{tabular}{c|c|c}
\hline \hline$|\mathcal{T}|(-)$ & $\mu_{e}\left({ }^{\circ} \mathrm{C}\right)$ & $t_{\phi}(-)$ \\
\hline $25 \times 25$ & 0.90 & 1397 \\
$50 \times 50$ & 0.52 & 4904 \\
$100 \times 100$ & 0.28 & 17352 \\
$150 \times 150$ & 0.19 & 36308 \\
\hline \hline
\end{tabular}

both approaches is further confirmed by Table I that lists the average absolute difference $\mu_{e}$ between $W\left(c_{i}, t_{\phi}\right)$ and the corresponding $T\left(x_{i}^{g}, y_{i}^{g}\right)$ together with $t_{\phi}$. As expected, it indicates that $\mu_{\phi}$ drops as we are working towards the continuum limit $|\mathcal{T}| \rightarrow \infty$. In order to verify the comparability between the results obtained using the CCA-based model on the one hand, and a finite difference approximation of Eq. (1) on the other hand, we implemented an implicit finite difference scheme for this equation, which was solved iteratively for $100 \times 100$ nodes located in the centers of gravity of the CCA's cells. As such, we assessed that the mean absolute difference between the simulated temperature of both approaches was only 0.01 , whereas the maximum discrepancy was 3.4 . This clearly indicates the comparability existing between both approaches, as such granting reliability to the approach we propose.

Figure 5 further shows that the regions in which the discrepancies between the simulation results obtained using the approaches at stake are the most pronounced, are located in the corners of the tessellated surface, whereas the smallest discrepancies can be found in the surface's center. These discrepancies can be attributed to the fact that $W\left(c_{i}, t+1\right)$ is calculated using the temperature values in cells that share an edge with $c_{i}$, as well as cells that merely share a vertex with $c_{i}$ since a Moore neighborhood is employed. As such, neighboring cells that are not directly facing $c_{i}$ have the same influence on the determination of $W\left(c_{i}, t+1\right)$ as the facing ones, which, physically speaking, would entail a flow of heat through $c_{i}$ 's four corner points that is equally large as the heat flow through its sides. In order to work around this artifact that is inevitably related to the use of a square tessellation, either the steady-state temperature distribution can be computed using Eq. (3) or one can give up the square tessellation and resort to a more intricate meshing method yielding cells which state can be iteratively calculated by means of Eq. (3). Yet, the first workaround is not more than a stopgap since then it is assumed that cells merely sharing a vertex with $c_{i}$ have no influence at all on $W\left(c_{i}, t+1\right)$, which is as doubtful as granting them equal importance as cells sharing an edge with $c_{i}$.

\section{The CCA-based model's strengths exemplified}

In the remainder of this section, we consider the flow of heat that originates from a tube (radius $0.4 \mathrm{~cm}$ ) conducting

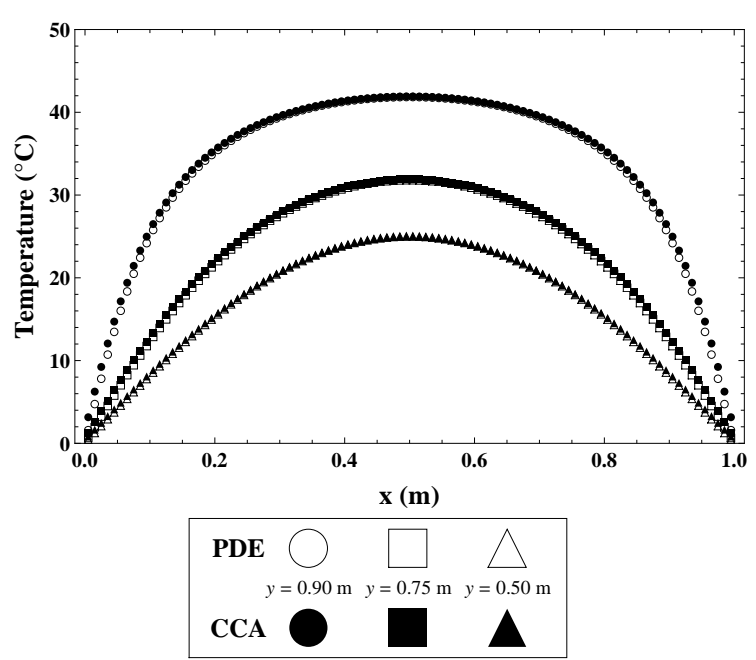

Fig. 4. Steady-state temperature distribution along three horizontal crosssections obtained using the analytical solution (Eq. (4)) on the one hand, and the CCA-based model (Eq. (2)), on the other hand.

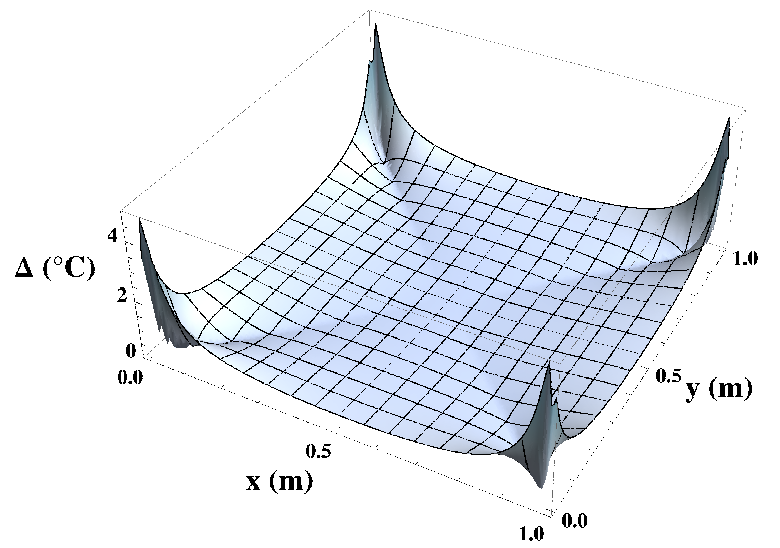

Fig. 5. Absolute difference, denoted $\Delta\left({ }^{\circ} \mathrm{C}\right)$, between $W\left(c_{i}, t_{\phi}\right)$ and the corresponding $T\left(x_{i}^{g}, y_{i}^{g}\right)$, where $t_{\phi}$ is the number of time steps needed such that $\left|W\left(c_{i}, t+1\right)-W\left(c_{i}, t\right)\right| \leq \phi, \forall c_{i} \in \mathcal{T}$ and $\left(x_{i}^{g}, y_{i}^{g}\right)$ represents $c_{i}$ 's center of gravity.

a fluid maintained at a temperature of $50^{\circ} \mathrm{C}$. This tube is homogeneously wrapped up with a lagging material and the wrapped tube is contained in a box of which the sides are maintained at a temperature of $20^{\circ} \mathrm{C}$. Seen the fact that the temperature of the fluid, as well as the sides of the box are maintained constant, it is to be expected that a steadystate temperature distribution will be reached inside the box after a sufficient amount of time. Obviously, the geometry of the surface that needs to tessellated, together with the circular boundary condition obstructs the use of a regular tessellation, though, a Delaunay tessellation can be employed for discretizing. Figure 6 depicts a transversal cross-section through this construction and shows the Delaunay tessellation, consisting of 12,688 cells, that was used to mesh the space enclosed between the box' sides and the tube.

After implementing the boundary conditions as indicated 


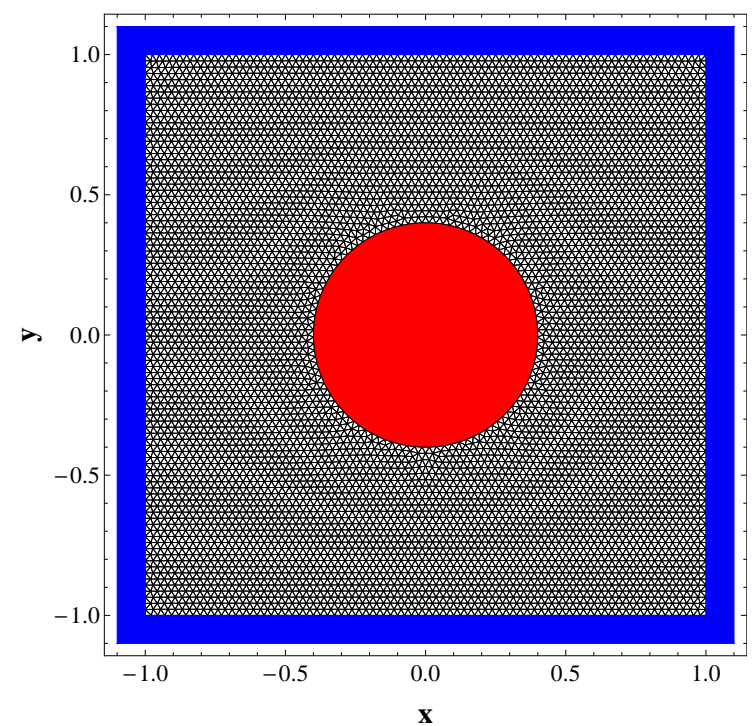

Fig. 6. Schematic overview of a transversal cross-section through a box containing a tube homogeneously wrapped up with lagging material. The sides of the box are maintained at $20^{\circ} \mathrm{C}$, while a warm fluid $\left(50^{\circ} \mathrm{C}\right)$ is flowing through the tube.

in Fig. 6, the steady-state temperature distribution in the lagfilled area was computed iteratively using Eq. (2) and is visualized in Fig. 7(a). In order to satisfy the halting criterion, 6,048 iterative evaluations of Eq. (2) were needed. Figure 7(b) depicts the temperature distribution along four crosssections through the medium. For comprehensiveness, we should emphasize that the latter figure was obtained by taking the values of the cells of which the center of gravity was located the closest to the equidistant points along each of the four cross-sections.

\section{Comparison with other numerical techniques}

Nonetheless we leave a thorough comparison between the results obtained using the above presented CCA-based model on the one hand, and well-established numerical techniques that have been developed for approximating the solution of the Laplace equation if intricate boundary conditions and/or geometries are involved on the other hand, to future work, we briefly place the former methodology against both the finite difference method (FDM) and the finite element method (FEM). Typically, the former method discretizes a PDE based upon a square tessellation of $\mathbb{R}^{2}$, which inevitably leads to the same difficulties as the ones facing when using the CCA-based model on such a tessellation. Besides, the central difference approximation of Eq. (1) collapses to Eq. (2) if the discretization size in both the $x$ - and $y$-direction are the same. With regard to the FEM, it is clear that it coincides with the CCA-based approach in the sense that it relies on the same meshing methods that are used by FEM, but setting up a sound finite element approximation of the problem at hand is certainly not as straightforward as implementing Eq. (2) or (3). Unavoidably, these equations yield an approximation of Eq. (1), but likewise, one may not lose sight of the fact that also FDM and FEM yield only an approximate solution of Eq. (1).

Unavoidably, a thorough comparison between the performance of the CCA-based models on the one hand and the FEM and FDM on the other hand will lead to the question whether either a continuous model or a discrete model is the most appropriate starting point for numerical modeling. To emphasize this dilemma, we may refer to Toffoli [37] who remarked already in 1984 that is questionable to stick to PDEs as a starting point for numerical modeling as soon as symbolic manipulations are no longer possible since (1) they represent a stylized form of the process(es) at stake, (2) we need to discretize them in order to find a numerical approximation, and (3) real-valued variables are rounded off. Perhaps, discrete models might offer a less roundabout way to model natural processes?

\section{CONCLUSION}

In this paper we established the mathematical constructs that are needed to make use of the irregular CCA paradigm to build models upon that can be of use in applied sciences, and we illustrated this paradigm's usability for a CCA-based model describing steady-state heat flow. Although we obtained the tessellation used throughout this paper by means of a well-established meshing method, it could be equally well deduced from a vector-based geographical information system that exploits irregular polygons as one of its three major data types, in addition to vertices and line segments, to encode spatial information.

\section{REFERENCES}

[1] M. Gardner, "Mathematical games: The fantastic combinations of John Conway's new solitaire game 'Life'," Scientific American, vol. 223, pp. 120-123, 1971.

[2] J. von Neumann, "The general and logical theory of automata," in Cerebral Mechanisms in Behaviour: The Hixon Symposium, L. Jeffress, Ed. New York, United States: Wiley, 1951, pp. 1-32.

[3] K. Kaneko, Ed., Theory and Applications of Coupled Map Lattices. Chichester, United Kingdom: John Wiley \& Sons Ltd., 1993.

[4] J. M. Baetens and B. De Baets, "Phenomenological study of irregular cellular automata based on Lyapunov exponents and Jacobians," Chaos, vol. 20, p. 033112, 2010.

[5] J. Jost and M. P. Joy, "Spectral properties and synchronization in coupled map lattices," Physical Review E, vol. 65, pp. 016201-1-016201-9, 2001.

[6] M. Mackey and J. Milton, "Asymptotic stability of densities in coupled map lattices," Physica D, vol. 80, pp. 1-17, 1995.

[7] R. J. Doran and S. W. Laffan, "Simulating the spatial dynamics of foot and mouth disease outbreaks in feral pigs and livestock in Queensland, Australia, using a susceptible-infected-recovered cellular automata model," Preventive Veterinary Medicine, vol. 70, pp. 133-152, 2005.

[8] S. H. White, A. M. del Rey, and G. R. Sanchez, "Modeling epidemics using cellular automata," Applied Mathematics and Computation, vol. 186, pp. 193-202, 2007.

[9] A. Alexandridis, D. Vakalis, C. Siettos, and G. Bafas, "A cellular automata model for forest fire spread prediction: The case of the wildfire that swept through Spetses Island in 1990," Applied Mathematics and Computation, vol. 204, pp. 191-201, 2008.

[10] S. G. Berjak and J. W. Hearne, "An improved cellular automaton model for simulating fire in a spatially heterogeneous savanna system," Ecological Modelling, vol. 148, pp. 133-151, 2002.

[11] L. H. Encinas, S. H. White, A. M.del Rey, and G. R. Sánchez, "Modelling forest fire spread using hexagonal cellular automata," Applied Mathematical Modelling, vol. 31, pp. 1213-1227, 2007. 


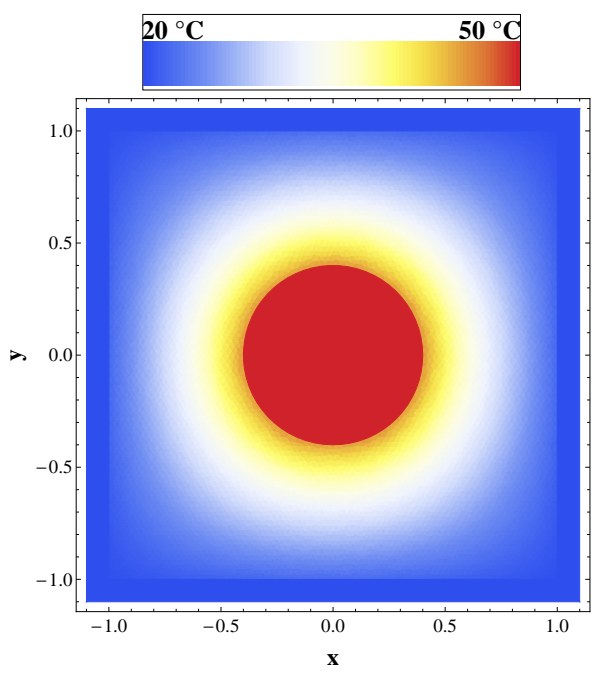

(a)

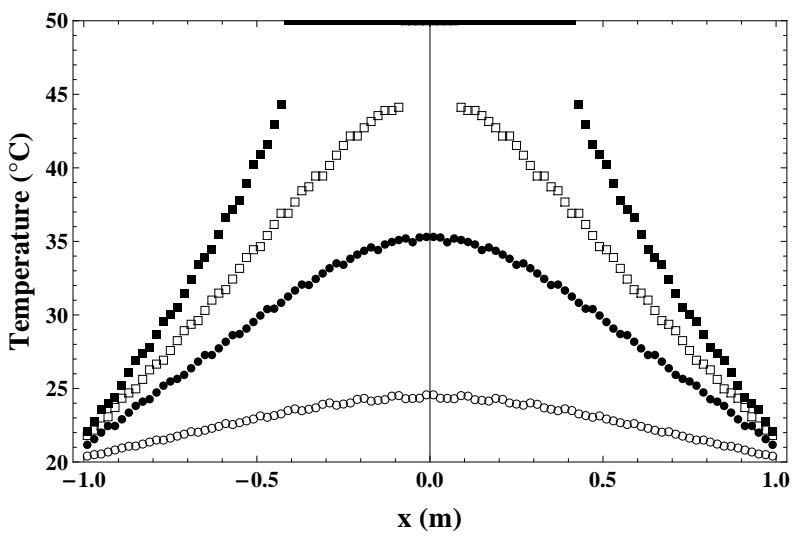

(b)

Fig. 7. Steady-state temperature distribution in the lag-filled area (a) and along four cross-sections through the medium (b).

[12] M. Batty, "Cellular dynamics: modelling urban growth as a spatial epidemic," in Geocomputational Modelling, M. M. Fischer and Y. Leung, Eds. Berlin, Germany: Springer, 2001, pp. 109-141.

[13] H. Baltzer, P. W. Braun, and W. Köhler, "Cellular automata models for vegetation dynamics," Ecological Modelling, vol. 107, pp. 113-125, 1998.

[14] C. Picioreanu, M. C. M. van Loosdrecht, and J. J. Heijnen, "Mathematical modeling of biofilm structure with a hybrid differential-discrete cellular automaton approach," Biotechnology and Bioengineering, vol. 58, pp. 101-116, 1998.

[15] G. Pizarro, D. Griffeath, and D. R. Noguera, "Quantitative cellular automaton model for biofilms," Journal of Environmental Engineering, vol. 127, pp. 782-789, 2001.

[16] D. G. Mallet and L. G. De Pillis, "A cellular automata model of tumorimmune system interactions," Journal of Theoretical Biology, vol. 239, pp. 334-350, 2006

[17] A. Qi, X. Zheng, C. Du, and B. An, "A cellular automaton model of cancerous growth," Journal of Theoretical Biology, vol. 161, pp. 1-12, 1993.

[18] B. Chopard, P. O. Luthi, and P. A. Queloz, "Cellular automata model of car traffic in a two-dimensional street network," Journal of Physics A-Mathematical and General, vol. 29, pp. 2325-2336, 1996.

[19] O. K. Tonguz, W. Viriyasitavat, and F. Bai, "Modeling urban traffic: A cellular automata approach," IEEE Communications Magazine, vol. 47, pp. 142-150, 2009.

[20] M. Fonstad, "Cellular automata as analysis and synthesis engines at the geomorphology-ecology interface," Geomorphology, vol. 77, pp. 217234, 2006.

[21] B. Carter, "Cellular automata on triangular, pentagonal and hexagonal tessellations," in Encyclopedia of Complexity and Systems Sciences, R. Meyers, Ed. Heidelberg, Germany: Springer, 2009.

[22] D. D'Ambrosio, S. Di Gregorio, and G. Iovine, "Simulating debris flows through a hexagonal cellular automata model: SCIDDICAS $_{3-h e x}$," Natural Hazards and Earth System Sciences, vol. 3, pp. 545-559, 2003.

[23] L. Berec, "Techniques of spatially explicit individual-based models: construction, simulation and mean-field analysis," Ecological Modelling, vol. 150 , pp. 55-81, 2002.

[24] M. Badoual, P. Derbez, M. Aubert, and B. Grammaticos, "Simulating the migration and growth patterns of Bacillus subtilis," Physica A, vol. 388, pp. 549-559, 2009.

[25] M. P. Coleman, An Introduction to Partial Differential Equations with MATLAB. Boca Raton, United States: Chapman \& Hall/CRC, 2005.

[26] G. Crisci, G. Iovine, S. D. Gregorio, and V. Lupiano, "Lava-flow hazard on the SE flank of Mt. Etna (Southern Italy)," Journal of Volcanology and Geothermal Research, vol. 177, pp. 778-796, 2008.

[27] D. D'Ambrosio and W. Spataro, "Parallel evolutionary modelling of geological processes," Parallel Computing, vol. 33, no. 3, pp. 186-212, 2007.

[28] D. Favis-Mortlock, "A self-organizing dynamic systems approach to the simulation of rill initiation and development on hillslopes," Computers and Geosciences, vol. 24, pp. 353-372, 1998.

[29] J. A. Parsons and M. A. Fonstad, "A cellular automata model of surface water flow," Hydrological Processes, vol. 21, pp. 2189-2195, 2007.

[30] A. Nishiyama, T. Tokihiro, M. Badoual, and B. Grammaticos, "Modelling the morphology of migrating bacterial colonies," Physica D, vol. 239, pp. 1573-1580, 2010.

[31] N. Fatés and M. Morvan, "Perturbing the topology of the game of life increases its robustness to asynchrony," Lecture Notes in Computer Science, vol. 3305, pp. 111-120, 2004.

[32] A. Flache and R. Hegselmann, "Do irregular grids make a difference? Relaxing the spatial regularity in cellular models in social dynamics," Journal of Artificial Societies and Social Simulation, vol. 4, 2001.

[33] W. Shi and M. Y. Cheung Pang, "Development of Voronoi-based cellular automata: an integrated dynamic model for geographical information systems," International Journal of Geographical Information Science, vol. 14, pp. 455-474, 2000.

[34] A. Ilachinski, Ed., Cellular Automata. A Discrete Universe. London, United Kingdom: World Scientific, 2001.

[35] S. Wolfram, A New Kind of Science. Champaign, United States: Wolfram Media Inc., 2002.

[36] J. L. Schiff, Cellular Automata: A Discrete View of the World. Chichester, United Kingdom: John Wiley \& Sons Ltd., 2008.

[37] T. Toffoli, "Cellular automata as an alternative to (rather than an approximation of) differential equations in modeling physics," Physica $D$, vol. 10 , pp. 117-127, 1984. 